\title{
Exciton generation and recombination dynamics of quantum dots embedded in GaNAsP nanowires
}

\author{
M. Jansson $\odot,{ }^{1, *}$ R. La, ${ }^{2}$ C. W. Tu, ${ }^{2}$ W. M. Chen,${ }^{1}$ and I. A. Buyanova ${ }^{1, *}$ \\ ${ }^{1}$ Department of Physics, Chemistry and Biology, Linköping University, SE-58183 Linköping, Sweden \\ ${ }^{2}$ Department of Electrical and Computer Engineering, University of California, San Diego, La Jolla, California 92093, USA
}

(Received 5 November 2020; revised 27 January 2021; accepted 8 March 2021; published 26 April 2021)

\begin{abstract}
Semiconductor quantum dots (QDs) acting as single-photon-emitters are potential building blocks for various applications in future quantum information technology. For such applications, a thorough understanding and precise control of charge states and capture/recombination dynamics of the QDs are vital. In this work, we study the dynamics of QDs spontaneously formed in GaNAsP nanowires, belonging to the dilute nitride material system. By using a random population model modified for these highly mismatched materials, we analyze the results from photoluminescence and photon correlation experiments and show a general trend of disparity in positive and negative trion populations and also a strong dependence of the capture/recombination dynamics and QD charge states on its surroundings. Specifically, we show that the presence of hole-trap defects in the proximity to some QDs facilitates formation of negative trions, which also causes a dramatic reduction of the neutral exciton lifetime. These findings underline the importance of proper understanding of the QD capture and recombination processes and demonstrate the possibility to use highly mismatched materials and defects for charge engineering of QDs.
\end{abstract}

DOI: 10.1103/PhysRevB.103.165425

\section{INTRODUCTION}

Optically bright semiconductor quantum dots (QDs) are, through their inherent highly efficient single-photon emission, one of the most promising building blocks for a wide range of novel optical devices [1], such as quantum logic gates [2,3], and optically controlled switches [4], which can be utilized in various quantum communications [5] and quantum computation schemes [6]. For these applications, optimizing exciton generation and recombination dynamics of a QD and its surroundings are of vital importance. For example, charges surrounding QDs may lead to deterioration of their performance, due to, e.g., a reduction of QD hole dephasing times [7], an intermittent luminescence [8], spectral diffusion [9], and perturbations of the exciton recombination that leads to additional exciton recombination pathways [10]. On the other hand, surrounding charges in the form of, e.g., impurity defects have been proposed [11] and recently utilized, e.g., optical switching [4], further demonstrating the pivotal effects of the QD surroundings on its dynamics.

On-chip integration of optical and electronic components and also fabrication of all-optical devices may be facilitated by implementing the QD in the nanowire (NW) geometry,

\footnotetext{
${ }^{*}$ Corresponding authors: mattias.jansson@liu.se; irina.bouianova@liu.se

Published by the American Physical Society under the terms of the Creative Commons Attribution 4.0 International license. Further distribution of this work must maintain attribution to the author(s) and the published article's title, journal citation, and DOI. Funded by Bibsam.
}

which can be easily integrated with a foreign material such as III-V NW grown on Si. Such geometry provides a certain directionality of the embedded QDs and allows for wave guiding of the emitted light [12], and enhanced light extraction efficiency [13,14]. Due to extensive research efforts, optically bright QDs have recently been realized in NWs made of conventional III-V materials, owing to their superior optical properties and mature growth techniques [15,16,17-25].

Most recently, growth of III-V NWs has been extended to dilute nitride alloys [26-29]. Dilute nitrides are known to have an unusual electronic structure and to experience a giant bowing in the bandgap energy due to a dramatic downshift of the conduction band (CB) upon the substitution of group-V host atoms by nitrogen [30] The bandgap bowing, combined with other changes of the electronic structure, largely increases flexibility in the bandgap engineering and makes these alloys attractive for a variety of applications in nanoelectronics and photonics [29]. Moreover, alloying with nitrogen in dilute nitride NWs has been found to result in spontaneous formation of optically-bright QDs caused by short-range fluctuations in the $\mathrm{N}$ composition [31-33]. We have demonstrated that the hole character in such QDs depends on the local strain and host alloy fluctuations [32,33], which opens up a possible avenue for valence state engineering in the QDs. Moreover, the QDs formed in GaNAsP NWs have most recently been shown to produce single photon emission [33], required for various applications in the field of quantum optics.

It is well known that the incorporation of $\mathrm{N}$ mainly affects the CB states [30]. Therefore, in dilute nitride-based QDs a three-dimensional confinement potential exists only for electrons while holes are expected to be mainly localized through a Coulomb interaction induced by the confined electron, which is different from QDs embedded in conventional 
III-V semiconductors and alloys. An in-depth understanding of generation and recombination processes in such QDs is currently lacking. Moreover, dilute nitride materials are prone to defect formation, which is facilitated by nonequilibrium growth conditions utilized for their fabrication. The influence of the formed defects on the properties of the QDs in the dilute nitride NWs is hitherto unknown.

In this work we address these issues by using continuous wave (cw) and transient photoluminescence (PL) and photon correlation techniques. We show that two types of QDs with distinctly different exciton dynamics and charge states coexist in GaNAsP NWs. Based on the random population model modified for the dilute nitrides, these two types are attributed to the QDs that are either isolated or interacting with nearby defect centers. Our results, therefore, underline a critical role of the local surrounding in generation and recombination processes in the QDs and show that defect engineering could constitute an important tool in engineering future quantum optical devices utilizing semiconductor QDs.

\section{RESULTS AND DISCUSSION}

The investigated GaNAsP NWs were grown by gas source molecular beam epitaxy (MBE) on a (111) Si substrate. A detailed description of the investigated samples and experimental details can be found in Methods. We have previously shown [33] that low-temperature micro-PL ( $\mu$ PL) spectra from such NWs contain numerous sharp lines, due to excitonic emissions from spontaneously formed QDs as a result of short-range fluctuations in the $\mathrm{N}$ composition. In this work, our detailed steady-state and time-resolved PL studies show that the formed QDs can be divided into two categories. The first category, which is exemplified by QD1, includes QDs showing only neutral exciton and biexciton emissions. Such QDs constitute about $60 \%$ of the studied QDs (20 in total). To the second category (exemplified by QD2) we assign QDs showing an additional trion emission.

\section{$Q D 1$}

Typical cw- $\mu$ PL spectra acquired from QD1 with varying excitation power $\left(\mathrm{W}_{\text {exc }}\right)$ are shown in Fig. 1(a). Two main peaks (labeled as X and XX) are observed, where $\mathrm{X}$ dominates the PL spectrum at low powers, while the XX gains its intensity at higher $\mathrm{W}_{\text {exc }}$. In Fig. 1(b), the integrated intensities of the $\mathrm{X}$ and $\mathrm{XX}$ peaks as a function of $\mathrm{W}_{\text {exc }}$ are shown (symbols) and exhibit linear and quadratic dependence, respectively. Moreover, according to polarization-resolved $\mu \mathrm{PL}$ measurements [see insets in Fig. 1(b)], each of the two lines consists of a pair of orthogonally polarized components, where the $\mathrm{X}$ and XX pairs show an opposite splitting of the equal magnitude $(120 \mu \mathrm{eV})$. All these properties are characteristic for biexciton (XX)-exciton (X) complexes in a QD [34]. Recombination dynamics of these lines was evaluated by performing time-resolved $\mu \mathrm{PL}$ measurements. The temporal profiles of the two PL lines are shown in Fig. 1(c) (the symbols). Both PL emission lines exhibit single exponential decays, and the $\mathrm{X}$ lifetime $(1.5 \mathrm{~ns})$ is significantly longer than that of $\mathrm{XX}$ (0.5 ns). This is consistent with the previous studies $[35,36]$ that shows that the $\mathrm{XX}$ lifetime can be $25-50 \%$ of the $\mathrm{X}$ lifetime, depending on the distributions of electron $(e)$ and hole $(h)$ wave functions within the QDs.

While the excitation-power dependent and polarizationresolved PL results make a strong case for the $\mathrm{X}$ and $\mathrm{XX}$ peak assignment, a definitive proof that the two lines originate from the same QD can be obtained from photon correlation (timecorrelated single-photon counting, TCSPC) measurements. Figures 1(d) and 1(e) present the measured autocorrelation histograms of the $\mathrm{X}$ and $\mathrm{XX}$ peak, respectively, while the $\mathrm{XX}, \mathrm{X}$ cross-correlation histogram is shown in Fig. 1(f) (the symbols). For $\mathrm{X}$, a clear antibunching at $t=0$ is observed testifying that the QD acts as a quantum emitter. For XX, strong photon bunching wings in the histogram are seen, which are caused by fast capture of an exciton after the biexciton recombination. Finally, in the cross-correlation measurement [Fig. 1(f)], where the detection of the XX (X) photon is used as a start (stop) signal for the correlation measurements, an antibunching-bunching behavior is seen, which is characteristic of the XX-X cascade recombination [37]. This unambiguously proves that $\mathrm{X}$ and $\mathrm{XX}$ stem from the same QD. From the PL peak separation [Fig. 1(a)], we find a biexciton binding energy of $E_{\mathrm{B}} \approx 1.35 \mathrm{meV}$.

\section{QD2}

We now investigate the second type of QDs, where besides the $\mathrm{X}$ and $\mathrm{XX}$ peaks a third $\left(\mathrm{X}^{*}\right)$ peak is observed in cw$\mu \mathrm{PL}$ spectra acquired using varying excitation powers - see Fig. 2(a). The integrated intensities of the three peaks are shown in Fig. 2(b). While the excitation power dependence of $\mathrm{XX}$ (orange spheres) is clearly superlinear with a power index of 1.68 , both $\mathrm{X}$ (blue triangles) and $\mathrm{X}^{*}$ (pink squares) show rather similar power dependences with the power indexes of 0.6 and 0.85 , respectively. According to the polarizationresolved PL measurements [shown in the insets in Fig. 2(b)], each of the $\mathrm{X}$ and $\mathrm{XX}$ peaks consists of a pair of orthogonally polarized lines with the opposite splitting of equal magnitude $(55 \mu \mathrm{eV})$ for $\mathrm{X}$ and $\mathrm{XX}$, similar to the $\mathrm{XX}-\mathrm{X}$ pair of QD1. The $\mathrm{X}^{*}$ peak, however, contains a single component that is weakly polarized (not shown here), as expected for charged excitons. The results of the time-resolved $\mu \mathrm{PL}$ measurements for the three lines are shown in Fig. 2(c). The X decay is found to be significantly accelerated as compared with that in the QD1. It exhibits a fast decay component during approximately 0.4 ns after the peak intensity, followed by a slow decay component (1.8 ns) that corresponds to the radiative lifetime of $\mathrm{X}$. Moreover, the fast decay of $\mathrm{X}$ coincides with a rise of the $X^{*}$ emission, which suggests that it is caused by the evolution of $\mathrm{X}$ into $\mathrm{X}^{*}$. Both $\mathrm{X}^{*}$ and XX PL decays are found to be single exponential, with a lifetime of 1.9 and $0.38 \mathrm{~ns}$, respectively.

Similar to QD1, we use the TCSPC spectroscopy to prove that the three peaks in Fig. 2(a) originate from the same QD. The cross-correlation histograms of the $X^{*}$ peak are shown in Figs. 2(d) $\left(X^{*}, X\right)$ and $2 \mathrm{e}\left(\mathrm{XX}, \mathrm{X}^{*}\right)$, whereas that for $(\mathrm{XX}, \mathrm{X})$ is shown in Fig. 2(f). In all cases, clear antibunching is observed at $t=0$ with a reasonable agreement between the measured $\mathrm{g}^{2}(0)$ value and that expected taking into account the uncorrelated background signal and the finite time-response of the detectors (the dotted horizontal line), as described in Methods. 

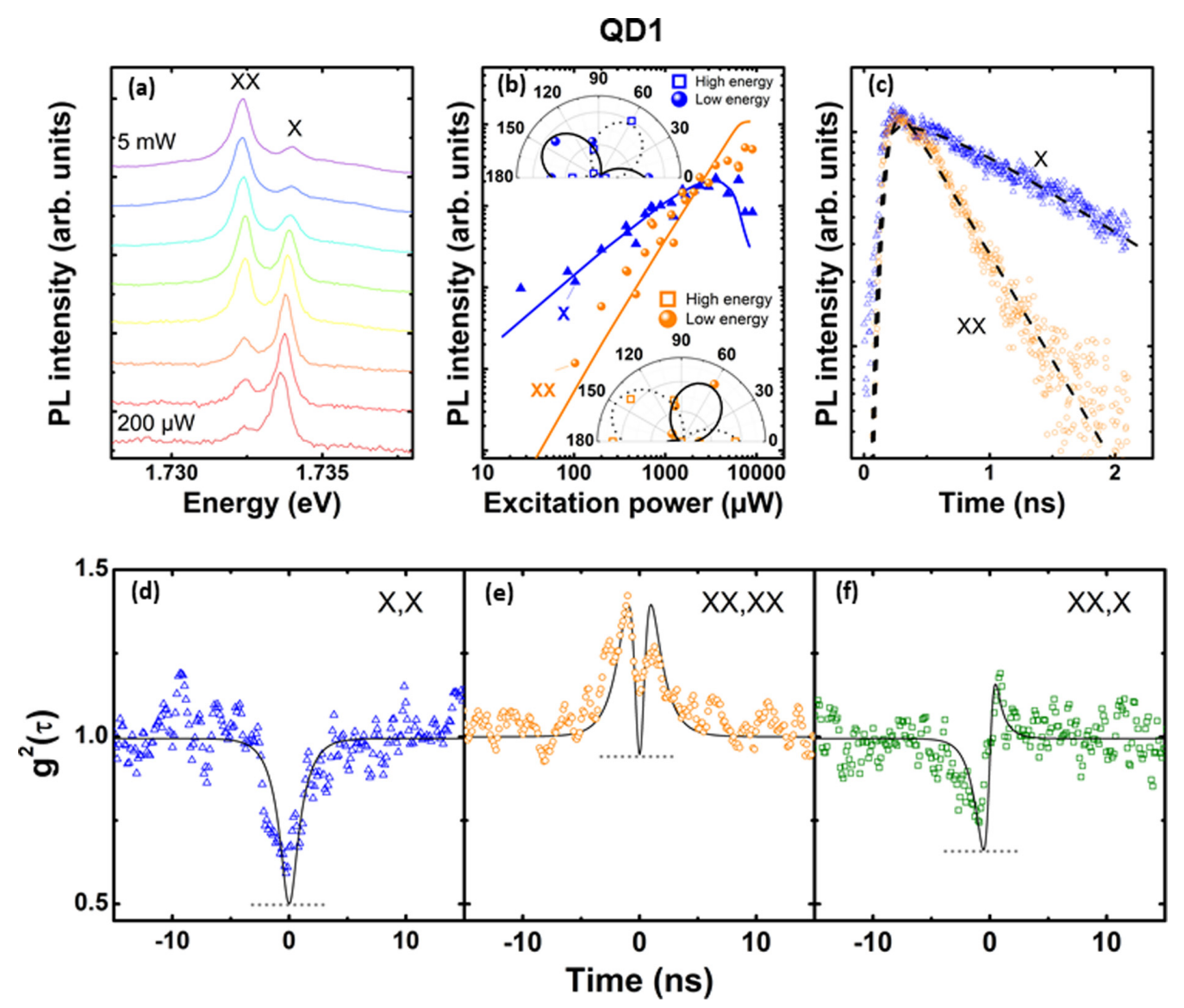

FIG. 1. Optical properties of QD1. (a) $\mu \mathrm{PL}$ spectra acquired from QD1 at different excitation powers. The spectra are normalized to the same maximum intensity and are shifted vertically for clarity. (b) Integrated intensities of X (the blue triangles) and XX (the orange spheres) at different excitation powers. The solid lines are the results of the RPM simulations. The insets show angular plots of the integrated PL intensity of the two polarization-resolved components of $X$ (the upper inset, blue symbols) and XX (the lower inset, orange symbols), measured by rotating the azimuthal angle $\varphi$ of a linear polarization analyzer placed before the monochromator. Here, $\varphi=0$ represents linear polarization detected parallel to the NW axis. The intensity has been corrected for the optical antenna effect, as described in Ref. [34]. (c) Normalized $\mu \mathrm{PL}$ transients of $\mathrm{X}$ (the blue triangles) and XX (the orange circles), after subtraction of the overlapping broad background signal. The black solid lines are RPM simulated results. (d)-(f): Results of photon correlation measurements: autocorrelation histograms of X (d) and XX (e), and cross-correlation histogram between XX (start) and X (stop) (f). The RPM simulation results, which take into account the uncorrelated background signal and are convoluted with a detector response function following the procedure described in Methods, are shown with the black solid lines. The expected value of $\mathrm{g}^{(2)}(0)$ is shown by the horizontal dotted lines. All simulations were performed using the parameters listed in Table II.

The TCSPC results, therefore, provide a direct experimental proof that the emission peaks indeed stem from excitonic complexes in the same QD.

From a comparison between the properties of QD1 and QD2 shown in Figs. 1 and 2, the following two main differences between these two types of QDs become apparent: (i) No $X^{*}$ emission can be observed in QD1 whereas it becomes strong in QD2, and (ii) in contrast to the slow decay of X seen in QD1, the X decay in QD2 contains the fast component that correlates with the rise of the $\mathrm{X}^{*}$ emission. To understand the revealed differences in the QD behavior, it is instructive to model the involved capture and recombination processes.

We model the QD system by using the so-called random population model (RPM) [38], which analyzes the probability of finding the QD in various charge configurations (or microstates), as shown schematically in Fig. 3. We only consider up to two-electron and two-hole charge configurations (i,j), where (i) and (j) refer to the numbers of electrons (e) and holes (h) in the QD that vary from 0 to 2 . In dilute nitride QDs, the electron confinement is caused by the downshift of the CB edge with increasing $\mathrm{N}$ content in localized areas. Since the valence band states are only weakly perturbed, the holes are instead bound by the Coulomb interaction with the electrons trapped in the QDs. For this reason, the QDs cannot be positively charged and the corresponding microstates are excluded from the analysis. In doing so, three exciton complexes are considered: the single exciton $\mathrm{X} \equiv(1,1)$, the biexciton $\mathrm{XX} \equiv$ $(2,2)$, and negatively charged trion $X^{-} \equiv(2,1)$.

The probability of finding the QD in a certain charge configuration $(i, j)$ is represented by the population $n_{i, j}$ of the corresponding microstate. For example, for an empty QD, the population of the microstate $(0,0)$ representing an empty QD is $n_{0,0}=1$, while all other $n_{i, j}=0$. The populations $n_{1,1}$, 

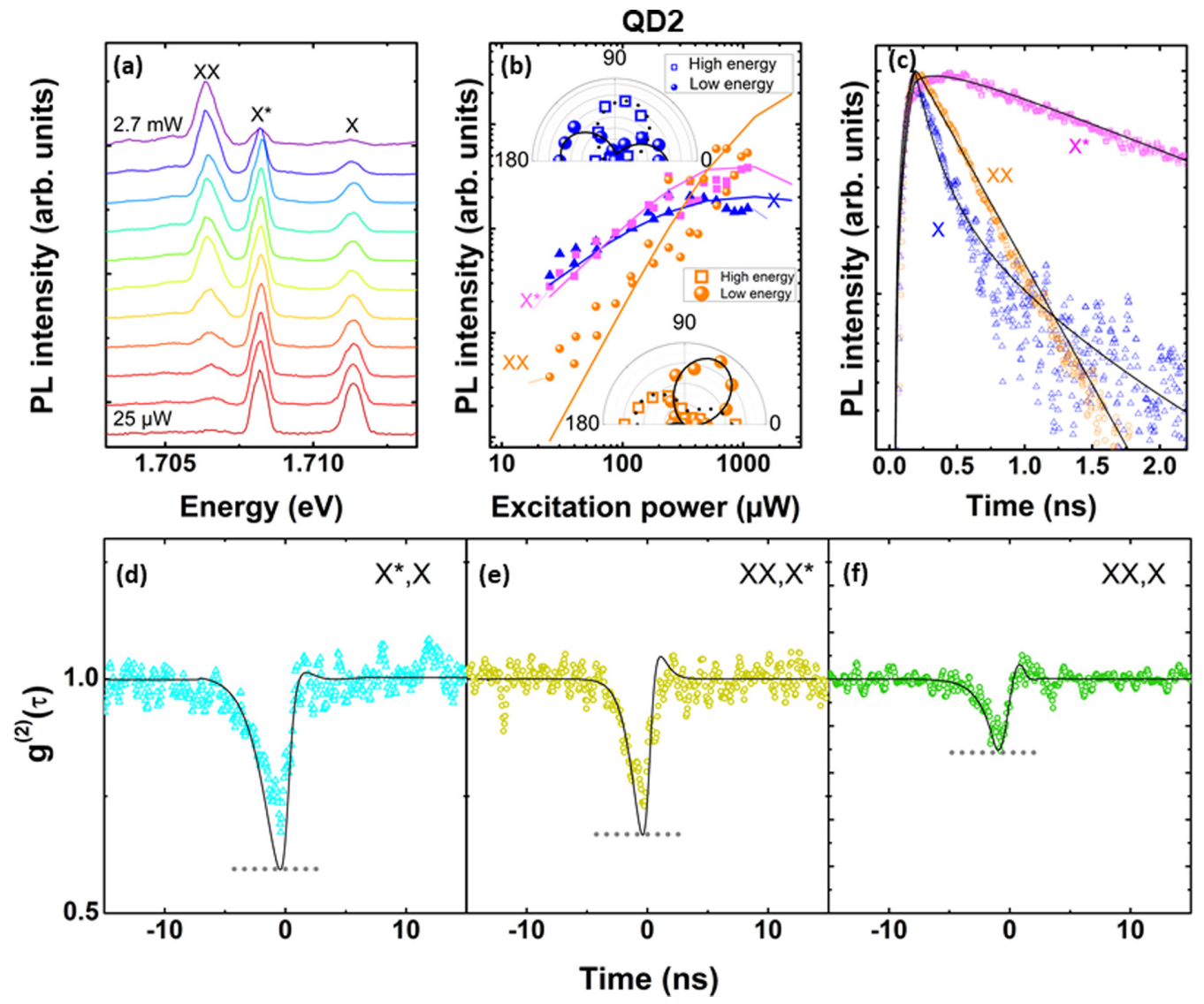

FIG. 2. Optical properties of QD2. (a) $\mu \mathrm{PL}$ spectra acquired from QD2 at different excitation powers. The spectra are normalized to the same maximum intensity and are shifted vertically for clarity. (b) Integrated intensities of $X$ (the blue triangles), $X^{*}$ (the pink squares) and XX (the orange spheres) at different excitation powers. The solid lines show the results of the RPM simulations. The insets show angular plots of the integrated PL intensity of the two polarization-resolved components of X (the upper inset, blue symbols) and XX (the lower inset, orange symbols), measured by rotating the azimuthal angle $\varphi$ of a linear polarization analyzer placed before the monochromator. Here, $\varphi=0$ represents linear polarization detected parallel to the NW axis. The intensity has been corrected for the optical antenna effect, as described in Ref. [34]. (c) Normalized $\mu \mathrm{PL}$ transients of $\mathrm{X}$ (the blue triangles), $\mathrm{X}^{*}$ (the pink squares) and XX (the orange circles), after subtraction of the overlapping broad background signal, shown together with the simulated curves (the solid lines) using the RPM. Cross-correlation histograms between $X^{*}$ (start) and X (stop) (d), XX (start) and X* (stop) (e), and XX (start) and X (stop) (f) peaks. The RPM simulation results, which take into account the uncorrelated background signal and are convoluted with a detector response function following the procedure described in Methods, are shown with the black solid lines. The expected value of $\mathrm{g}^{(2)}(0)$ is shown by the horizontal dotted lines. All simulations were performed using the parameters listed in Table II.

$n_{2,1}$, and $n_{2,2}$, of the three microstates corresponding to the three excitonic complexes $\mathrm{X}, \mathrm{X}^{-}$, and $\mathrm{XX}$, respectively, scale with their PL intensity [38]. (Since positive trions are not expected to form in the dilute nitride QD, the emission line labeled as $\mathrm{X}^{*}$ in Fig. 2 is suggested to correspond to $\mathrm{X}^{-}$). Besides the QD microstates, the model includes reservoirs of free electrons, holes, and neutral excitons, as shown in the lower part of Fig. 3. These reservoirs represent charge carriers/excitons within the extended states surrounding the single QD. Their respective populations $N_{e}, N_{h}$, and $N_{X}$ are governed by the optical generation with the rates of $G_{e-h}$ (electrons, holes) and $G_{X}$ (excitons), captured by the QD with the rates $C_{i, j}^{X, e, h}$ as defined below, and the recombination in the reservoir, represented by the coefficients $\alpha_{e-h}$ (for electrons and holes) and the rate $\alpha_{X}$ (for excitons). The reservoir charge carriers may also be captured by defects acting as traps or NR recombination channels, with capture coefficients $T_{e, h}^{D}$ (electrons, holes).
In dark, the QD is empty and is in the $(0,0)$ state, which will be converted to other microstates upon capture of photogenerated charge carriers/excitons. This is illustrated by the arrows in the upper part of Fig. 3, where the capture of an electron, a hole or an exciton is represented by the dotted, dashed, and solid lines, respectively. Radiative recombination processes with the characteristic recombination times, $\tau_{X}, \tau_{X-}$, and $\tau_{X X}$ reduce the $n_{1,1}, n_{2,1}$, and $n_{2,2}$ microstate populations, as is indicated by the wavy arrows. The sum of all microstate populations is always 1 . The capture and recombination processes of the modelled QD can be described by the following rate equations:

$$
\begin{aligned}
& \frac{d n_{i, j}}{d t}=C_{i, j}^{X} N_{X}+C_{i, j}^{e} N_{e}+C_{i, j}^{h} N_{h}+R_{i, j}, \\
& \frac{d N_{X}}{d t}=-N_{X}\left(\sum_{i, j} D_{i, j} C_{i, j}^{X}+\alpha_{X}\right)+G_{X},
\end{aligned}
$$




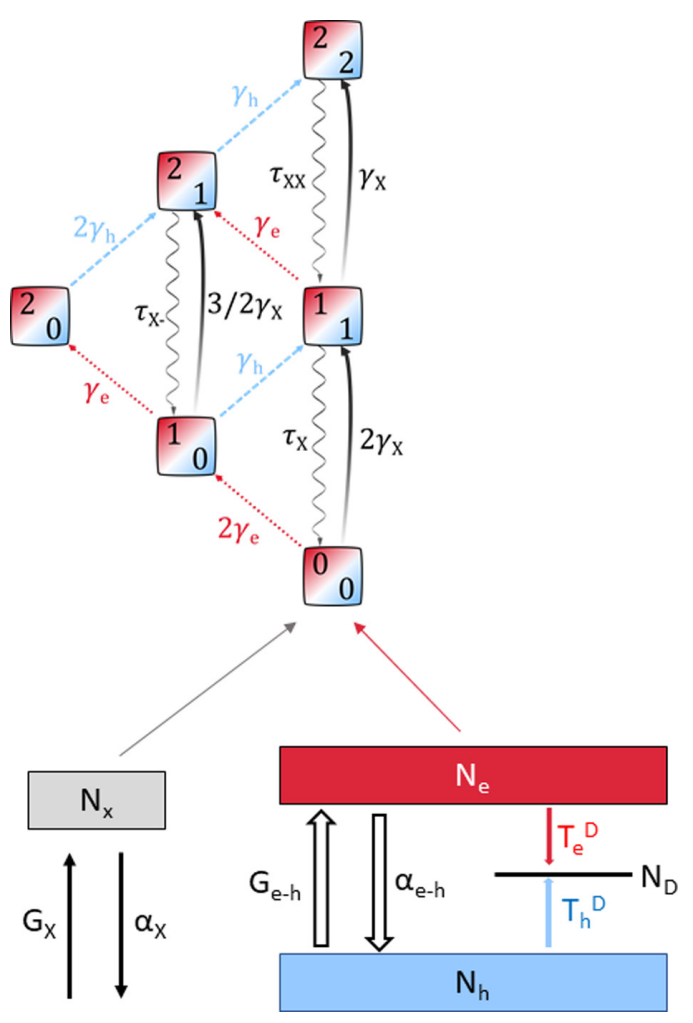

FIG. 3. Sketch of the generation, capture, and recombination dynamics in the employed model. The squares in the upper part of the figure represent the (i), $(j)$ charge states of the QD, where the numbers (i) and (j) refer to the number of captured electrons and holes, respectively. The dotted $\left(\gamma_{e}\right)$, dashed $\left(\gamma_{h}\right)$ and solid $\left(\gamma_{\mathrm{X}}\right)$ arrows illustrate electron, hole, and exciton capture, respectively, while the prefactors define the relative probabilities of the capture events. The wavy arrows $\left(\tau_{\mathrm{X}, \mathrm{X}-, \mathrm{XX}}\right)$ represent radiative recombination of the various excitonic complexes in the QD. The boxes in the lower part of the figure illustrate generation and recombination of free excitons $\left(N_{\mathrm{X}}\right)$, electrons $\left(N_{\mathrm{e}}\right)$ and holes $\left(N_{\mathrm{h}}\right)$ in the reservoirs, in the presence of a defects. All parameters are defined in the text.

$$
\begin{gathered}
\frac{d N_{e}}{d t}=-N_{e}\left(\sum_{i, j} i C_{i, j}^{e}+N_{h} \alpha_{e-h}\right) \\
-T_{e}^{D} N_{e}\left(N_{D}^{T}-N_{D}\right)+G_{e-h}, \\
\frac{d N_{h}}{d t}=-N_{h}\left(\sum_{i, j} j C_{i, j}^{h}+N_{e} \alpha_{e-h}\right)-T_{h}^{D} N_{h} N_{D}+G_{e-h}, \\
\frac{d N_{D}}{d t}=T_{e}^{D} N_{e}\left(N_{D}^{T}-N_{D}\right)-T_{h}^{D} N_{h} N_{D}, \\
\sum_{i, j} i n_{i, j}+N_{e}+N_{D}-N_{D}^{0}=\sum_{i, j} j n_{i, j}+N_{h} .
\end{gathered}
$$

Here $D_{i, j}$ is the minimum value of $i$ and $j . N_{D}^{T}$ is the total number of the prominent defect in carrier trapping and recombination, and $N_{D}$ is the number of defects occupied by an electron, with $N_{D}^{0}$ being its value in dark. The capture $C_{i, j}$ and recombination $R_{i, j}$ parameters are given in Table I. $\gamma_{(e, h, X)}$ are the capture coefficients of electrons, holes and excitons by the QD.
TABLE I. Capture $C_{i, j}$ and recombination $R_{i, j}$ parameters for each $(i, j)$ configuration of the QD.

\begin{tabular}{lcccc}
\hline \hline$(i, j)$ & $C_{i, j}^{X}$ & $C_{i, j}^{e}$ & $C_{i, j}^{h}$ & $R_{i, j}$ \\
\hline$(0,0)$ & $-2 \gamma_{X} n_{0,0}$ & $-2 \gamma_{e} n_{0,0}$ & 0 & $\frac{n_{1,1}}{\tau_{X}}$ \\
$(1,0)$ & $-\frac{3}{2} \gamma_{X} n_{1,0}$ & $2 \gamma_{e} n_{0,0}-\gamma_{e} n_{1,0}$ & $-\gamma_{h} n_{1,0}$ & $\frac{n_{2,1}}{\tau_{X-}}$ \\
$(2,0)$ & 0 & $\gamma_{e} n_{1,0}$ & $-2 \gamma_{h} n_{2,0}$ & 0 \\
$(1,1)$ & $2 \gamma_{X} n_{0,0}-\gamma_{X} n_{1,1}$ & $-\gamma_{e} n_{1,1}$ & $\gamma_{h} n_{1,0}$ & $-\frac{n_{1,1}}{\tau_{X}}+\frac{n_{2,2}}{\tau_{X X}}$ \\
$(2,1)$ & $\frac{3}{2} \gamma_{X} n_{1,0}$ & $\gamma_{e} n_{1,1}$ & $2 \gamma_{h} n_{2,0}-\gamma_{h} n_{2,1}$ & $-\frac{n_{2,1}}{\tau_{X-}}$ \\
$(2,2)$ & $\gamma_{X} n_{1,1}$ & 0 & $\gamma_{h} n_{2,1}$ & $-\frac{n_{2,2}}{\tau_{X X}}$ \\
\hline \hline
\end{tabular}

In the RPM, the time evolution of the various microstate, reservoir, and defect populations are obtained numerically, by initiating the QD in the empty microstate and letting the system evolve until convergence [39]. Both pulsed and cw generation may be used in the simulations. In the former case, the generation terms are implemented by Kronecker $\delta$ functions. Simulating the results of photon correlation experiments using the RPM is a two-step process. Firstly, the steady-state values of the reservoir and defect populations are found as described above. Secondly, keeping these values fixed, evolution of the chosen microstate populations is monitored. For example, in the $\mathrm{X}, \mathrm{X}$ autocorrelation experiment, the start and stop signals are the detection of a photon resulting from recombination of X. Consequently, after the emission of the first photon (the start signal), the QD is empty. The time delay until the second photon emission (the stop signal) depends on the repopulation of the $(1,1)$ charge configuration. In the corresponding simulation, at $t=0$ the microstate populations are set to $n_{0,0}=1$ and $n_{i, j}=0$ for all other $i, j$, and then the evolution of the $n_{1,1}$ is monitored.

Using the modified RPM, it is possible to single out the dominant factors that affect the QD properties. Since one of the main differences between QD1 and QD2 is the appearance of the trion emission, we first model the expected ratio of the $n_{2,1} / n_{1,1}$ microstate populations in dilute nitride QDs. For the case when $N_{D}^{T}=0$ and under cw excitation, simulations predict that $n_{2,1} / n_{1,1}<1$, as shown in Fig. 4(a). Clearly, the formation of the negative trion requires excess free electrons as compared to holes in the reservoir, either through the generation of more electrons than holes, which is unlikely under our experimental conditions of band-to-band light absorption, or removal of free holes from the reservoir. The latter can be modeled by introducing a hole-attractive defect with $T_{h}^{D}>T_{e}^{D}$. As expected, the $n_{2,1} / n_{1,1}$ ratio increases with an increasing number of such defects, whereas an opposite trend is predicted for an electron-attractive defect with $T_{h}^{D}<T_{e}^{D}$ [Fig. 4(a)]. The above simulations predict the existence of two types of QDs. One is dominated by X and XX recombination under the conditions that there is no defect (or with a concentration too low to compete with the QD in carrier capture) or only electron trapping defects in the vicinity of the $\mathrm{QD}$, whereas the other is dominated by $\mathrm{X}, \mathrm{XX}$, and $\mathrm{X}^{-}$recombination when the defects near the QD are predominantly hole traps. This prediction is in excellent agreement with our experimental observation of QD1 and QD2. The simultaneous 

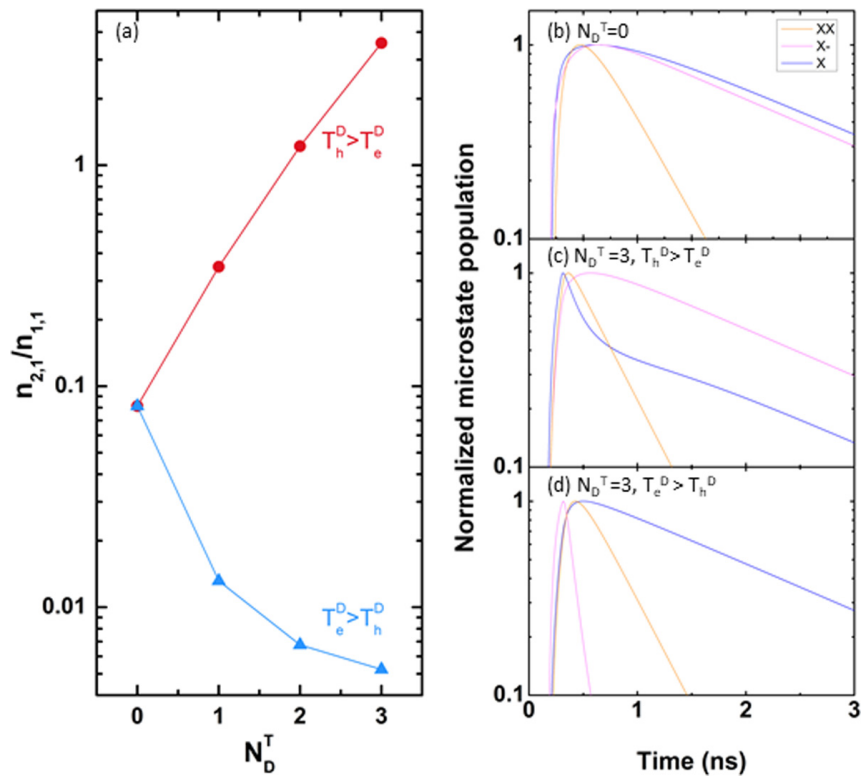

FIG. 4. Effects of defects nearby the QD. (a) Ratio between the $n_{2,1}$ and $n_{1,1}$ microstate populations for different numbers of electronattractive (the blue triangles) and hole-attractive (the red circles) defects and under continuous generation. (b)-(d) Dynamics of the microstate populations representing the $\mathrm{X}, \mathrm{X}^{-}$and $\mathrm{XX}$ microstates under pulsed generation, with $N_{D}^{T}=0$ (b) and $N_{D}^{T}=3$ (c),(d), where $T_{h}^{D}>T_{e}^{D}$ in (c) and $T_{e}^{D}>T_{h}^{D}$ in (d). The simulated decays are normalized to the same maximum intensity.

presence of both types of QDs is not surprising in view of a very short diffusion length for carriers/excitons that is characteristic for dilute nitrides with severe localization effects [40], such that each QD only experiences its own immediate surrounding within a highly localized area.

The changes in the QD charge state populations shown in Fig. 4(a) are also predicted to affect dynamics of the excitonic transitions. This can be seen from Figs. 4(b)-4(d), where the simulated transient behavior of the $n_{1,1}, n_{2,1}$, and $n_{2,2}$ microstate populations under the conditions of $N_{D}^{T}=0$ [Fig. 4(b)], $N_{D}^{T}=3, T_{h}^{D}>T_{e}^{D}$ [Fig. 4(c)] and $N_{D}^{T}=3, T_{h}^{D}<$ $T_{e}^{D}$ [Fig. 4(d)] are shown. The simulations were performed assuming $G_{X}=0, \gamma_{e}=\gamma_{h}=0.007 \mathrm{ps}^{-1}, \alpha_{e-h}=0.02 \mathrm{ps}^{-1}$, $N_{D}^{0}=0.5 N_{D}^{T}$. The defect capture coefficients used for the hole (electron) attractive defects are $T_{e(h)}^{D}=0.0005 \mathrm{ps}^{-1}, T_{h(e)}^{D}=$ $0.017 \mathrm{ps}^{-1}$. These parameters are relevant to the experimental conditions as will be justified below. Under conditions when no defects exist close to the QD [see Fig. 4(b)], single exponential decays determined by $\tau_{X}, \tau_{X-}$, and $\tau_{X X}$ are seen for the $n_{1,1}, n_{2,1}$, and $n_{2,2}$ populations, respectively. The presence of an electron-attractive defect speeds up the decay of the $n_{2,1}$ population [Fig. 4(d)]. On the other hand, introducing capture by the hole-attractive defect accelerates the $n_{1,1}$ decay [Fig. 4(c)]. Interestingly, this fast initial decay closely correlates with the rise of $n_{2,1}$, suggesting that the initial $n_{1,1}$ decay is caused by an additional electron capture soon after the generation pulse, which would increase the $n_{2,1}$ population at the expense of $n_{1,1}$. This observation can be explained as follows. At the time of the generation pulse $\left(t_{0}\right), N_{e}=N_{h}$. For $t>t_{0}$, the holes are quickly captured by the hole-attractive defect, leading to $N_{e}>N_{h}$, promoting the capture of an additional electron at the QD. At $t \gg t_{0}$, when both the populations $N_{e}$ and $N_{h}$ are small such that the conversion from X to $\mathrm{X}^{-}$ can no longer compete with $\mathrm{X}$ recombination, the $\mathrm{X}$ decay is dominated by the slow radiative recombination.

The results of the RPM simulations show that the presence of a hole-attractive defect near the QD can qualitatively explain the experimentally observed differences between the studied QD1 and QD2. By performing fitting of the experimental data with the aid of the RPM, a quantitative description of the capture and recombination processes may be obtained. To reduce the number of fitting parameters, the radiative lifetimes of the QD excitonic complexes were taken from the time-resolved PL data discussed above. The generation rate was chosen to match the simulated ratio of the $n_{1,1}$ and $n_{2,2}$ microstate populations to the measured ratio between the $\mathrm{X}$ and XX emission peaks. The best fit to the data was obtained using the parameters shown in Table II, while the simulation results are shown by the solid lines in Figs. 1 and 2. The simulations of the photon correlation $g^{(2)}$ functions are scaled taking into account the uncorrelated background of the experimental data, and are convoluted with the response function of the detector, which explains why $g^{(2)}(0)>0$. We see that an identical set of fitting parameters (given in Table II) can explain the experimental data for QD1 and QD2, except for the presence of hole-attractive defects in the vicinity to QD2, with the parameters also listed in Table II.

At present, the exact chemical origin of the hole-attractive defect nearby QD2 is unknown. From Table II, we can see that the capture coefficient $T_{h}{ }^{D}$ is more than one order of magnitude larger than $T_{e}{ }^{D}$, suggesting a large asymmetry in the capture cross sections for electrons and holes as expected for a hole trap. In addition, the defect should be abundant in dilute nitrides as $40 \%$ of the studied QDs belong to the QD2 category. It is well known that because of the low growth temperature and the presence of nitrogen in the alloy, dilute nitrides are prone to formation of various point defects [41]. For example, Ga vacancy $\left(V_{\mathrm{Ga}}\right)$ and related complexes are known to be common grown-in defects formed during MBE growth of $\mathrm{Ga}(\mathrm{N}, \mathrm{P}) \mathrm{As}$ NWs [42]. In undoped $\mathrm{Ga}(\mathrm{N}) \mathrm{As}, V_{\mathrm{Ga}}$ was shown to be in the 3- charge state in thermal equilibrium [43], which could capture photogenerated holes. $\mathrm{C}_{\mathrm{As}}$, a

TABLE II. Fitting parameters used for the RPM simulations of the experimental data presented in Figs. 1 and $2 . G_{\text {Tot }}$ is the sum of all generation rates, $G_{\mathrm{Tot}}=G_{e-h}+G_{X}$. RPM parameters for the defect center obtained from the fitting to the data.

\begin{tabular}{cccccccccc}
\hline \hline & $G_{X} / G_{\mathrm{Tot}}$ & $\gamma_{X}\left(\mathrm{ps}^{-1}\right)$ & $\gamma_{e}\left(\mathrm{ps}^{-1}\right)$ & $\gamma_{h}\left(\mathrm{ps}^{-1}\right)$ & $\alpha_{X}\left(\mathrm{ps}^{-1}\right)$ & $\alpha_{e-h}\left(\mathrm{ps}^{-1}\right)$ & $N_{D}^{T}$ & $N_{D}^{0}$ & $T_{e}^{D}\left(\mathrm{ps}^{-1}\right)$ \\
\hline QD1 & 0.2 & 0.01 & 0.007 & 0.1 & 0.3 & 0.02 & 0 & $T_{h}^{D}\left(\mathrm{ps}^{-1}\right)$ \\
QD2 & 0.2 & 0.01 & 0.007 & 0.1 & 0.3 & 0.02 & 3 & 3 & 0.0005 \\
\hline \hline
\end{tabular}


common residual impurity in MBE-grown GaAs-based materials, could also capture photogenerated holes when it is in the negative charge state in dark. Future research efforts are required to definitively identify the defect responsible for the charge tuning of the QDs.

\section{SUMMARY}

In summary, by employing $\mathrm{cw}$ and time-resolved $\mu \mathrm{PL}$ spectroscopy and photon correlation measurements, we show that two types of QDs with significantly different photoluminescence characteristics are spontaneously formed in dilute nitride GaNAsP NWs grown by MBE. Specifically, the first type shows single exciton and biexciton emission, whereas the second type also exhibits a strong emission from charged exciton recombination. Our simulations using the modified RPM successfully model these two types of QDs, determined by the unusual confinement potentials of the QDs in such dilute nitride NWs, unseen in QDs based on other conventional semiconductor systems. The simulations also satisfactorily explain the experimentally observed differences in capture and recombination processes of both types of the QDs by taking into account the absence and presence of hole-attractive defects in the vicinity of these QDs. It is concluded that the interaction with such defects can significantly alter the capture and recombination dynamics of QDs, and their charge states. This work demonstrates the feasibility of charge tuning of the QDs by utilizing defects, and it underlines the importance of the proper understanding and control of the QD surroundings for potential applications in optoelectronics and quantum optics.

\section{METHODS}

Self-catalyzed growth of the GaNAsP NWs was performed on (111) Si substrates using plasma-assisted MBE. $\mathrm{As}_{2}$ and $\mathrm{P}_{2}$ were supplied using thermally cracked $\mathrm{AsH}_{3}$ and $\mathrm{PH}_{3}$, respectively. Ga was supplied from a solid Ga source and $\mathrm{N}$ from an RF plasma. The NW growth was catalyzed using Ga droplets. Full details of the growth can be found in Ref. [28]. The NWs are found to be quite uniform in length $(2 \mu \mathrm{m})$ and diameter $(90 \mathrm{~nm})$. The $\mathrm{P}$ and $\mathrm{N}$ fraction in the group- $\mathrm{V}$ sublattice was $24 \%$ and $0.1 \%$, respectively [44].

The $\mu \mathrm{PL}$ spectroscopy and TCSPC experiments were performed in the backscattering geometry, using a continuous flow cryostat to cool down the sample to $6 \mathrm{~K}$. The same QDs in individual NWs, transferred to another Si substrate, were investigated in all experiments. For cw excitation, a solid-state 532-nm laser was used, while the $\mu \mathrm{PL}$ transient measurements were carried out using a pulsed Ti:Sapphire laser with a repetition rate of $76 \mathrm{MHz}$ and a pulse width of $150 \mathrm{fs}$. The laser light was focused on a NW to a spot below
$1 \mu \mathrm{m}$ in diameter by a $50 \times(0.5 \mathrm{NA})$ objective lens that was also used to collect the emission. In the cw and time-resolved $\mu \mathrm{PL}$ experiments, the emitted light was dispersed in a singlegrating monochromator and detected using a charged-coupled device (CCD) and streak camera, respectively. For the photon correlation experiments, a Hanbury-Brown and Twiss [45] interferometer was used, and the PL emission was guided into two different single-grating monochromators by a 50/50 beam splitter. The PL was detected using two avalanche photodiodes (APDs) with an estimated time resolution of at least $0.7 \mathrm{~ns}$, which strongly depends on the spot size. The time difference between the detection events of the two APDs was recorded using a TCSPC module.

In the analysis of $\mathrm{cw}$ and time-resolved $\mu \mathrm{PL}$ and TCSPC studies of the sharp QD lines, contributions from a spectrally overlapping and broad background emission have been subtracted. This broad PL band arises from recombination of weakly localized excitons, typical for dilute nitrides [46]. Its origin is distinctly different from that of the sharp QD lines emissions based on the following experimental findings: (i) the broad background and sharp line emissions exhibit different polarization properties [31]; (ii) thermal quenching of the sharp PL lines is substantially faster as compared with that of the broad background [32]; (iii) spatial distributions are different between them as revealed by the cathodoluminescence studies [33]. Their different origin is also confirmed by the shorter decay time of the sharp PL lines revealed in the present study. In the $\mu \mathrm{PL}$ data, the emission background spectrally immediately adjacent to the concerned sharp PL line was used as the background signal. Its $\mathrm{cw}$ intensity (or transient PL decay) was then subtracted from the corresponding data obtained by monitoring both the sharp lines and the broad background to retrieve the relevant background signal specific to the sharp lines. In the analysis of the photon correlation results, the simulated $\mathrm{g}^{(2)}$ functions are scaled to take into account the uncorrelated background as $\mathrm{g}_{\text {scaled }}^{(2)}=\rho^{2}\left(\mathrm{~g}^{(2)}-1\right)+$ 1 , where $\rho$ is the ratio of the QD and total PL intensities. The $\mathrm{g}_{\text {scaled }}^{(2)}$ function is then convoluted with the response function of the setup: a Gaussian function with full width at half maximum of 700 ps. From this, the expected value of $g^{(2)}(0)$, taking into account the uncorrelated background signal and the detector response, is obtained and shown by the horizontal dotted lines in Figs. 1 and 2.

\section{ACKNOWLEDGMENTS}

The authors acknowledge the financial support from the Swedish Research Council (Grant No. 2019-04312). I.B. and W.M.C. acknowledge financial support from the Swedish Government Strategic Research Area in Materials Science on Functional Materials at Linköping University (Faculty Grant SFO-Mat-LiU No. 2009 00971).

The authors declare no competing financial interest.
[1] I. Aharonovich, D. Englund, and M. Toth, Solid-State singlephoton emitters, Nat. Photonics 10, 631 (2016).

[2] M. Pooley, D. J. P. Ellis, R. B. Patel, A. J. Bennett, K. H. A. Chan, I. Farrer, D. A. Ritchie, and A. J. Shields, Controlled-
NOT gate operating with single photons, Appl. Phys. Lett. 100, 211103 (2012).

[3] Y. M. He, Y. He, Y. J. Wei, D. Wu, M. Atatüre, C. Schneider, S. Höfling, M. Kamp, C. Y. Lu, and J. W. Pan, On-Demand 
semiconductor single-photon source with near-unity indistinguishability, Nat. Nanotechnol. 8, 213 (2013).

[4] D. Rivas, G. Muñoz-Matutano, J. Canet-Ferrer, R. GarcíaCalzada, G. Trevisi, L. Seravalli, P. Frigeri, and J. P. MartínezPastor, Two-Color single-photon emission from InAs quantum dots: Toward logic information management using quantum light, Nano Lett. 14, 456 (2014).

[5] H. K. Lo, M. Curty, and K. Tamaki, Secure quantum key distribution, Nat. Photonics 8, 595 (2014).

[6] P. Kok, W. J. Munro, K. Nemoto, T. C. Ralph, J. P. Dowling, and G. J. Milburn, Linear optical quantum computing with photonic qubits, Rev. Mod. Phys. 79, 135 (2007).

[7] A. Greilich, S. G. Carter, D. Kim, A. S. Bracker, and D. Gammon, Optical control of one and two hole spins in interacting quantum dots, Nat. Photonics 5, 702 (2011).

[8] M. Sugisaki, H. W. Ren, K. Nishi, and Y. Masumoto, Fluorescence intermittency in self-assembled InP quantum dots, Phys. Rev. Lett. 86, 4883 (2001).

[9] A. Högele, S. Seidl, M. Kroner, K. Karrai, R. J. Warburton, B. D. Gerardot, and P. M. Petroff, Voltage-Controlled Optics of a Quantum Dot, Phys. Rev. Lett. 93, 217401 (2004).

[10] A. Lemaître, A. D. Ashmore, J. J. Finley, D. J. Mowbray, M. S. Skolnick, M. Hopkinson, and T. F. Krauss, Enhanced phononassisted absorption in single InAs/GaAs quantum dots, Phys. Rev. B. 63, 161309(R) (2001).

[11] S. Moskalenko, V. Donchev, K. F. Karlsson, P. O. Holtz, B. Monemar, V. Schoenfeld, M. Garcia, and M. Petroff, Effect of an additional infrared excitation on the luminescence efficiency of a single InAs/GaAs quantum dot, Phys. Rev. B. 68, 155317 (2003)

[12] R. Yan, D. Gargas, and Y. Peidong, Nanowire photonics, Nat. Photonics 3, 569 (2009).

[13] M. E. Reimer, G. Bulgarini, N. Akopian, M. Hocevar, M. B. Bavinck, M. A. Verheijen, E. P. A. M. Bakkers, L. P. Kouwenhoven, and V. Zwiller, Bright single-photon sources in bottom-up tailored nanowires, Nat. Commun. 3, 737 (2012).

[14] J. Claudon, J. Bleuse, N. S. Malik, M. Bazin, P. Jaffrennou, N. Gregersen, C. Sauvan, P. Lalanne, and J. M. Gérard, A highly efficient single-photon source based on a quantum dot in a photonic nanowire, Nat. Photonics 4, 174 (2010).

[15] M. T. Björk, C. Thelander, A. E. Hansen, L. E. Jensen, M. W. Larsson, L. R. Wellenberg, and L. Samuelson, Few-Electron quantum dots in nanowires, Nano Lett. 4, 1621 (2004).

[16] M. T. Borgström, V. Zwiller, E. Müller, and A. Imamoglu, Optically bright quantum dots in single nanowires, Nano Lett. 5, 1439 (2005).

[17] T. Shi, H. E. Jackson, L. M. Smith, N. Jiang, Q. Gao, H. H. Tan, C. Jagadish, C. Zheng, and J. Etheridge, Emergence of localized states in narrow GaAs/AlGaAs nanowire quantum well tubes, Nano Lett. 15, 1876 (2015)

[18] E. D. Minot, F. Kelkensberg, M. van Kouwen, J. A. van Dam, L. P. Kouwenhoven, V. Zwiller, M. T. Borgström, O. Wunnicke, M. A. Verheijen, and E. P. A. M. Bakkers, Single quantum dot nanowire LEDs, Nano Lett. 7, 367 (2007).

[19] J. Renard, R. Songmuang, C. Bougerol, B. Daudin, and B. Gayral, Exciton and biexciton luminescence from single GaN/AIN quantum dots in nanowires, Nano Lett. 8, 2092 (2008)

[20] J. Heinrich, A. Huggenberger, T. Heindel, S. Reitzenstein, S. Höfling, L. Worschech, and A. Forchel, Single photon emis- sion from positioned GaAs/AlGaAs photonic nanowires, Appl. Phys. Lett. 96, 211117 (2010)

[21] E. Uccelli, J. Arbiol, J. R. Morante, and A. Fontcuberta i Morral, InAs quantum dot arrays decorating the facets of GaAs nanowires, ACS Nano 4, 5985 (2010).

[22] N. Akopian, G. Patriarche, L. Liu, J. C. Harmand, and V. Zwiller, Crystal phase quantum dots, Nano Lett. 10, 1198 (2010).

[23] K. A. Dick, C. Thelander, L. Samuelson, and P. Caroff, Crystal phase engineering in single InAs nanowires, Nano Lett. 10, 3494 (2010).

[24] M. Heiss, B. Ketterer, E. Uccelli, J. R. Morante, J. Arbiol, and A. Fontcuberta i Morral, In(Ga)As quantum dot formation on group-III assisted catalyst-free InGaAs nanowires, Nanotechnology 22, 195601 (2011).

[25] X. Yan, X. Zhang, X. Ren, H. Huang, J. Guo, X. Guo, M. Liu, Q. Wang, S. Cai, and Y. Huang, Growth of InAs quantum dots on gaas nanowires by metal organic chemical vapor deposition, Nano Lett. 11, 3941 (2011).

[26] Y. Araki, M. Yamaguchi, and F. Ishikawa, Growth of dilute nitride GaAsN/GaAs heterostructure nanowires on Si substrates, Nanotechnology 24, 065601 (2013).

[27] Y. J. Kuang, S. Sukrittanon, H. Li, and C. W. Tu, Growth and photoluminescence of self-catalyzed GaP/GaNP core/shell nanowires on $\mathrm{Si}(111)$ by gas source molecular beam epitaxy, Appl. Phys. Lett. 100, 053108 (2012).

[28] R. La, J. L. Pan, F. Bastiman, and C. W. Tu, Selfcatalyzed $\mathrm{Ga}(\mathrm{N})$ AsP nanowires and GaAsP/GaNAsP core-shell nanowires grown on $\mathrm{Si}$ (111) by gas-source molecular beam epitaxy, J. Vac. Sci. Technol. B 34, 02L108 (2016).

[29] I. A. Buyanova and W. M. Chen, Dilute nitrides-based nanowires - a promising platform for nanoscale photonics and energy technology, Nanotechnology 30, 292002 (2019)

[30] W. Walukiewicz, W. Shan, K. M. Yu, J. W. Ager, E. E. Haller, I. Miotkowski, M. J. Seong, H. Alawadhi, and A. K. Ramdas, Interaction of Localized Electronic States with the Conduction Band: Band Anticrossing in II-VI Semiconductor Ternaries, Phys. Rev. Lett. 85, 1552 (2000).

[31] S. Filippov, M. Jansson, J. E. Stehr, J. Palisaitis, P. O. A. Persson, F. Ishikawa, W. M. Chen, and I. A. Buyanova, Strongly polarized quantum-dot-like light emitters embedded in GaAs/GaNAs core/shell nanowires, Nanoscale 8, 15939 (2016).

[32] M. Jansson, F. Ishikawa, W. M. Chen, and I. A. Buyanova, N-Induced Quantum Dots in GaAs/Ga(N, As) Core/Shell Nanowires: Symmetry, Strain, and Electronic Structure, Phys. Rev. Appl. 10, 044040 (2018).

[33] M. Jansson, L. Francaviglia, R. La, C. W. Tu, W. M. Chen, and I. A. Buyanova, Formation, electronic structure, and optical properties of self-assembled quantum-dot single-photon emitters in $\mathrm{Ga}(\mathrm{N}, \mathrm{As}, \mathrm{P})$ nanowires, Phys. Rev. Mater. 4, 056005 (2020).

[34] R. Seguin, A. Schliwa, S. Rodt, K. Potschke, U. W. Pohl, and D. Bimberg, Size-Dependent Fine-Structure Splitting in Self-Organized InAs/GaAs Quantum Dots, Phys. Rev. Lett. 95, 257402 (2005).

[35] P. A. Dalgarno, J. M. Smith, J. McFarlane, B. D. Gerardot, K. Karrai, A. Badolato, P. M. Petroff, and R. J. Warburton, Coulomb interactions in single charged self-assembled 
quantum dots: Radiative lifetime and recombination energy, Phys. Rev. B. 77, 245311 (2008).

[36] M. Sahin and F. A. Koc, A model for the recombination and radiative lifetime of trions and biexcitons in spherically shaped semiconductor nanocrystals, Appl. Phys. Lett. 102, 183103 (2013).

[37] E. Moreau, I. Robert, L. Manin, V. Thierry-Mieg, J. M. Gérard, and I. Abram, Quantum cascade of photons in semiconductor quantum dots, Phys. Rev. Lett. 87, 183601 (2001).

[38] M. Grundmann and D. Bimberg, Theory of random population for quantum dots, Phys. Rev. B 55, 9740 (1997).

[39] J. Gomis-Bresco, G. Muñoz-Matutano, J. Martínez-Pastor, B. Alén, L. Seravalli, P. Frigeri, G. Trevisi, and S. Franchi, Random population model to explain the recombination dynamics in single InAs/GaAs quantum dots under selective optical pumping, New J. Phys. 13, 023022 (2011).

[40] U. Jahn, S. Dhar, R. Hey, O. Brandt, J. Miguel-Sánchez, and A. Guzmán, Influence of localization on the carrier diffusion in GaAs (Al,Ga)As and (In,Ga)(As,N) GaAs quantum wells: A comparative study, Phys. Rev. B 73, 125303 (2006).
[41] Physics and Applications of Dilute Nitrides, I. A. Buyanova, W. M. Chen, editors (Taylor \& Francis, London, 2004).

[42] J. E. Stehr, S. L. Chen, M. Jansson, F. Ishikawa, W. M. Chen, and I. A. Buyanova, Defect formation in GaAs/GaNxAs1-x core/shell nanowires, Appl. Phys. Lett. 109, 203103 (2016).

[43] F. El-Mellouhi and N. Mousseau, Self-vacancies in gallium arsenide: An ab initio calculation, Phys. Rev. B. 71, 125207 (2005).

[44] M. Jansson, S. Chen, R. La, J. E. Stehr, C. W. Tu, W. M. Chen, and I. A. Buyanova, Effects of nitrogen incorporation on structural and optical properties of GaNAsP nanowires, J. Phys. Chem. C 121, 7047 (2017).

[45] R. Hanbury Brown and R. Q. Twiss, Correlation between Photons in Two Coherent Beams of Light, Nature (London) 177, 27 (1956).

[46] I. A. Buyanova, W. M. Chen, G. Pozina, J. P. Bergman, B. Monemar, H. P. Xin, and C. W. Tu, Mechanism for low-temperature photoluminescence in GaNAs/GaAs structures grown by molecular-beam epitaxy, Appl. Phys. Lett. 75, 501 (1999). 